\title{
Computed tomography scan can be misleading in the lateralisation of aldosterone excess
}

\author{
G Leksic1, A M Alduk², V Molnar1, A Haxhiu1, A Haxhiu1, A Balasko1, N Knezevic³, \\ T Dusek ${ }^{4}$ and D Kastelan 4
}

1Division of Endocrinology and Diabetes, Department of Internal Medicine, 2Department of Radiology, University Hospital Centre Zagreb, Zagreb, Croatia, ${ }^{3}$ Department of Urology, and ${ }^{4}$ Division of Endocrinology and Diabetes, Department of Internal Medicine, University Hospital Centre Zagreb, School of Medicine, University of Zagreb, Zagreb, Croatia

Correspondence should be addressed to G Leksic

Email

gleksic@gmail.com

\section{Summary}

Primary aldosteronism (PA) is characterised by aldosterone hypersecretion and represents a common cause of secondary hypertension. During diagnostic evaluation, it is essential to determine the aetiology of PA since the treatment of unilateral and bilateral disease differs significantly. Adrenal vein sampling (AVS) has been implemented as a gold standard test for the diagnosis of PA subtype. However, due to the AVS complexity, costs and limited availability, many patients with PA are being treated based on the computed tomography (CT) findings. In this article, we present two patients with discrepant CT and AVS results, demonstrating that AVS is the only reliable method for localising the source of aldosterone excess.

\section{Learning points:}

- $\mathrm{CT}$ is an unreliable method for distinguishing aldosterone-producing adenoma (APA) from bilateral adrenal hyperplasia (BAH).

- CT can be misleading in defining lateralisation of the aldosterone excess in case of unilateral disease (APA).

- AVS is the gold standard test for defining the PA subtype.

\section{Background}

Primary aldosteronism (PA) is the most common cause of endocrine hypertension (1). It is related to significant cardiovascular morbidity and should be diagnosed and treated promptly (1). Appropriate treatment of PA depends upon the correct classification: whether it is unilateral or bilateral aldosterone hypersecretion (2). The usage of CT in defining aetiology of PA is controversial and, due to a rather high prevalence of adrenal incidentaloma in general population, it can be misleading in many patients $(3,4)$. The gold standard for establishing the aetiology of
PA is adrenal vein sampling (AVS) (2). However, AVS is expensive, technically demanding and its availability is therefore quite limited (4). Nevertheless, due to the lack of availability of AVS, diagnosis and further treatment decisions for patients with PA are sometimes based on the results of the CT scan (3).

In this article, we present two cases of discrepant results of CT imaging and AVS which demonstrate that CT is not a reliable method for localising the source of excess aldosterone production. 


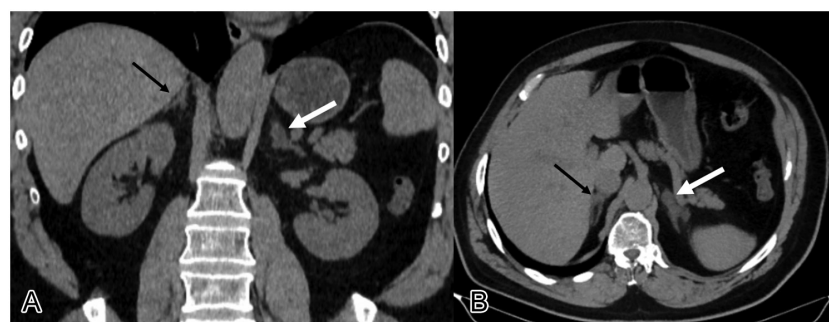

Figure 1

Case 1. Coronal (A) and axial (B) nonenhanced CT scan of the abdomen showing tumour on the left adrenal gland (white arrow). Right adrenal gland is without abnormalities (black arrow).

\section{Case 1}

\section{Case presentation}

A 59-year-old male was referred to the emergency department for severe headache. His personal history was uneventful, except for arterial hypertension over the last 5 years. Recently, the blood pressure was inadequately controlled, despite the continuous therapy with the combination of indapamide and ramipril. His BMI was $30 \mathrm{~kg} / \mathrm{m}^{2}$ and blood pressure was 195/115 $\mathrm{mmHg}$. He had no renal bruits and no signs of the Cushing's syndrome. A laboratory analysis showed marked hypokalaemia of $2.4 \mathrm{mmol} / \mathrm{L}$.

\section{Investigation}

Ambulatory 24-h BP monitoring revealed $>82 \%$ of readings above normal. A hormonal workup, performed after antihypertensive treatment was stopped for 2 weeks, showed increased aldosterone-to-renin ratio (ARR) without aldosterone suppression in the saline infusion test (SIT; $2 \mathrm{~L}$ of intravenous $0.9 \%$ saline over $4 \mathrm{~h}$ ). Adrenal CT demonstrated a $13 \mathrm{~mm}$ hypodense nodule in the left adrenal gland, whereas the right adrenal gland appeared normal (Fig. 1). In contrast, AVS demonstrated aldosterone hypersecretion in the right gland with the lateralisation index of 13.1 and the contralateral suppression index of 0.05 (Table 1).

\section{Treatment, outcome and follow-up}

Laparoscopic adrenalectomy of the right adrenal gland was performed and histology was consistent with small adrenocortical adenoma. Follow-up evaluation, performed 3 months after the surgery, revealed a well-controlled BP (without any antihypertensive treatment), a normal potassium level and adequate aldosterone suppression after SIT (Table 1).

\section{Case 2}

\section{Case presentation}

A 62-year-old female was referred for the evaluation of hypokalaemia (serum potassium concentration: $2.0 \mathrm{mmol} / \mathrm{L}$ ) that was discovered during a workup for acute cholecystitis. Her BMI was $27.8 \mathrm{~kg} / \mathrm{m}^{2}$ and blood

Table 1 Hormonal workup in case 1.

Parameters at diagnosis of PA
PRA ( $\mu \mathrm{g} / \mathrm{L} / \mathrm{h})$
PAC (pmol/L)
ARR
Post SIT PAC (pmol/L)
AVS results
Left adrenal vein
Right adrenal vein
Inferior vena cava
Lateralisation index
Contralateral suppression Index
Follow-up (3 months after surgery)
Post SIT PAC (pmol/L)
Potassium (mmol/L)
Average BP (mmHg)

\begin{tabular}{c}
\hline Values \\
\hline $0.1(0.2-2.8)$ \\
$1267(20-410)$ \\
$457(<30)$ \\
$1060(<277)$
\end{tabular}

$\overline{\text { Aldosterone }(\mathrm{pmol} / \mathrm{L})}$

Cortisol (nmol/L) A/C

\begin{tabular}{crrr} 
& 4860 & 34,960 & 0.13 \\
& 42100 & 23,040 & 1.82 \\
13.1 & 2420 & 1036 & 2.33 \\
0.05 & & & \\
& & & \\
83 & & & \\
4.1 & & & \\
$128 / 80$ & & & \\
\hline
\end{tabular}

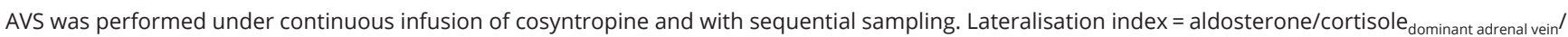
aldosterone/cortisole ${ }_{\text {non-dominant adrenal vein. }}$ Lateralisation index $>4$ was used for defining lateralisation of aldosterone excess. Contralateral suppression index = aldosterone/cortisol non-dominant adrenal vein $_{\text {/aldosterone/cortisole }}$ inf.vena cava.

PRA (plasma renin activity). PAC (plasma aldosterone concentration). Post SIT PAC (post saline infusion test PAC) $>277$ pmol/L was used as a criterion for the diagnosis of PA. 


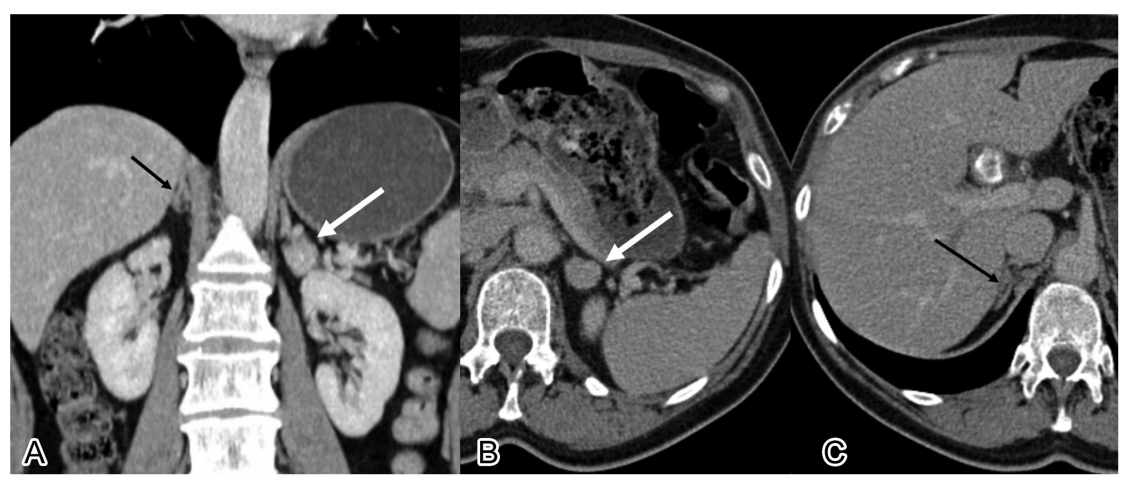

\section{Figure 2}

Case 2. Coronal (A) and axial (B and C) contrast-enhanced CT scan of the abdomen showing tumour on the left adrenal gland (white arrow). Right adrenal gland is without abnormalities (black arrow).

pressure was $180 / 100 \mathrm{mmHg}$. Her personal history revealed arterial hypertension, diagnosed at the age of 38 years and gallstones. She has been taking valsartan and lercanidipine, which were discontinued 2 weeks prior to hormonal evaluation.

\section{Investigation}

Raised ARR with no PAC (plasma aldosterone concentration) suppression after the saline infusion test led to the diagnosis of PA. CT demonstrated a $19 \mathrm{~mm}$ nodule in the left adrenal gland (Fig. 2), whereas AVS lateralised aldosterone hypersecretion in the right adrenal gland (Table 2).

\section{Treatment, outcome and follow-up}

Laparoscopic adrenalectomy of the right adrenal gland was performed and histology was consistent with small adrenocortical adenoma. A follow-up evaluation performed 3 months after the surgery confirmed remission of the disease with normal serum potassium concentration and well-controlled blood pressure with one antihypertensive drug (lercanidipine) (Table 2).

\section{Discussion}

PA represents an autonomous aldosterone hypersecretion that can originate from one or both adrenal glands. In $60 \%$ of cases, the underlying aetiology of PA is bilateral adrenal

Table 2 Hormonal workup in case 2.

\begin{tabular}{l}
\hline \\
\hline Parameters at diagnosis of PA \\
PRA $(\mu \mathrm{g} / \mathrm{L} / \mathrm{h})$ \\
PAC $(\mathrm{pmol} / \mathrm{L})$ \\
ARR \\
Post SIT PAC (pmol/L) \\
AVS results \\
Left adrenal vein \\
Right adrenal vein \\
Inferior vena cava \\
Lateralisation index \\
Contralateral suppression Index \\
Follow-up (3 months after surgery) \\
Post SIT PAC (pmol/L) \\
Potassium (mmol/L) \\
Average BP (mmHg)
\end{tabular}

\begin{tabular}{c}
\hline Values \\
\hline $0.1(0.2-2.8)$ \\
$947(20-410)$ \\
$341(<30)$ \\
$516(<277)$
\end{tabular}

Aldosterone (pmol/L)

Cortisol (nmol/L)

AVS was performed under continuos infusion of cosyntropine and with sequential sampling. Lateralisation index = aldosterone/cortisole dominant adrenal vein $^{\prime}$

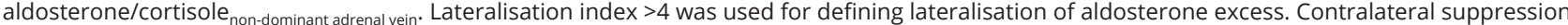
index $=$ aldosterone/cortisol non-dominant adrenal vein $_{\text {/aldosterone/cortisole }}$ inf.vena cava.

PRA (plasma renin activity). PAC (plasma aldosterone concentration). Post SIT PAC (post saline infusion test PAC) $>277$ pmol/L was used as a criterion for the diagnosis of PA. 
hyperplasia (BAH). In 35\% of cases, it is aldosteroneproducing adenoma (APA), whereas other causes, such as unilateral adrenal hyperplasia and aldosteroneproducing adrenocortical carcinoma, account for about $5 \%$ of patients $(1,2)$. However, from the clinical point of view, it is crucial to separate bilateral from unilateral aldosterone hypersecretion as the treatment of the two differs significantly.

According to current guidelines, AVS is the gold standard test for establishing the aetiology of PA with sensitivity and specificity of 95 and 100\%, respectively (2). However, AVS is an expensive, invasive, complex procedure, without standardised criteria and available only in a limited number of specialised centres (3, 4). Therefore, young patients (aged $<35$ years) with spontaneous hypokalaemia, marked aldosterone excess and unilateral adrenal mass, may not need AVS before proceeding to unilateral adrenalectomy (2).

CT represents another option for determining the source of aldosterone excess, but its overall accuracy in defining PA aetiology is less than $60 \%(5,6)$ due to the fact that some aldosteronomas are small tumours which might not be detected on CT scans. Moreover, due to a large prevalence of adrenal incidentaloma among the general population, a nonfunctional incidentaloma can be easily misdiagnosed for APA (5).

Many authors, in order to distinguish BAH from APA, have developed scoring systems based on the combination of clinical, biochemical and radiological parameters $(6,7)$. In accordance with said systems, the non-peformance of AVS may be considered in patients scoring results suggestive of BAH (7). Nevertheless, in patients with unilateral disease, the scoring system does not provide the information on the side of the affected gland. Accordingly, both of our patients would have been appropriately diagnosed with unilateral disease, but surgical treatment based on the CT scan would have resulted in the removal of the unaffected adrenal gland.

The necessity to perform AVS was also questioned in the SPARTACUS trial (8), which was the first randomised diagnostic trial in this clinical area. The study consisted of PA patients randomly assigned to undergo either CT or AVS in order to define the PA subtype and associated treatment. Follow-up was performed after 1 year and no significant difference was found in the clinical outcome between the two groups; the CT-based treatment was equally effective as the AVS-based treatment in terms of antihypertensive medication and health-related quality of life (8).
Nevertheless, the validity of the study results was quite limited by significant selection bias and a rather inappropriate choice of the primary endpoint for this type of study (9). Furthermore, regarding the biochemical remission of the disease, there was a trend in favour of AVS ( $80 \%$ biochemical remission in the CT versus $89 \%$ in the AVS group) (8). It is possible that, with a sufficient number of patients, that trend would have reached significance. A recently published large retrospective study comparing the diagnostic value of CT and AVS for unilateral PA reported favourable biochemical outcomes of adrenalectomy in PA patients whose surgical management was based on AVS (10). They concluded how patients diagnosed only by CT had a decreased likelihood of achieving complete biochemical success post surgery (10).

In this article, we presented two patients with PA caused by unilateral disease. In both cases, CT showed a tumour on the left side, whereas AVS lateralised aldosterone hypersecretion on the opposite side. These two patients demonstrate that even in the case of a high probability of unilateral disease, identification of the adrenal gland responsible for the aldosterone hypersecretion should be based exclusively on the results of AVS.

\section{Declaration of interest}

The authors declare that there is no conflict of interest that could be perceived as prejudicing the impartiality of the research reported.

\section{Funding}

This research did not receive any specific grant from any funding agency in the public, commercial or not-for-profit sector.

\section{Patient consent}

Written informed consent for publication has been obtained from the patient.

\section{Author contribution statement}

Leksic Gloria wrote the first draft and all authors contributed to the editing process. All authors were involved in the direct care of the patient.

\section{References}

1 Young WF Jr, Calhoun DA, Lenders JWM, Stowasser M \& Textor SC. Screening for endocrine hypertension: an Endocrine Society scientific statement. Endocrine Reviews 201738 103-122. (https://doi. org/10.1210/er.2017-00054)

2 Funder JW, Carey RM, Mantero F, Murad MH, Reincke M, Shibata H, Stowasser M \& Young WF Jr. The management of primary aldosteronism: case detection, diagnosis, and treatment: 
an Endocrine Society clinical practice guideline. Journal of Clinical Endocrinology and Metabolism 2016101 1889-1916. (https://doi. org/10.1210/jc.2015-4061)

3 Rossi GP, Barisa M, Allolio B, Auchus RJ, Amar L, Cohen D, Degenhart C, Deinum J, Fischer E, Gordon R, et al. The adrenal vein sampling international study (avis) for identifying the major subtypes of primary aldosteronism. Journal of Clinical Endocrinology and Metabolism 201297 1606-1614. (https://doi.org/10.1210/ jc.2011-2830)

4 Stewart PM \& Allolio B. Adrenal vein sampling for primary aldosteronism: time for a reality check. Clinical Endocrinology 201072 146-148. (https://doi.org/10.1111/j.1365-2265.2009.03714.x)

5 Young WF. Primary aldosteronism: renaissance of a syndrome. Clinical Endocrinology 200766 607-618. (https://doi.org/10.1111/ j.1365-2265.2007.02775.x)

6 Lim V, Guo Q, Grant CS, Thompson GB, Richards ML, Farley DR \& Young WF Jr. Accuracy of adrenal imaging and adrenal venous sampling in predicting surgical cure of primary aldosteronism. Journal of Clinical Endocrinology and Metabolism 201499 2712-2719. (https://doi.org/10.1210/jc.2013-4146)
7 Kupers EM, Amar L, Raynaud A, Plouin PF \& Steichen O. A clinical prediction score to diagnose unilateral primary aldosteronism. Journal of Clinical Endocrinology and Metabolism 201297 3530-3537. (https://doi.org/10.1210/jc.2012-1917)

8 Dekkers T, Prejbisz A, Kool LJS, Groenewoud HJMM, Velema M, Spiering W, Kołodziejczyk-Kruk S, Arntz M, Kądziela J, Langenhuijsen JF, et al. Adrenal vein sampling versus CT scan to determine treatment in primary aldosteronism: an outcome-based randomised diagnostic trial. Lancet: Diabetes and Endocrinology 20164 739-746. (https://doi.org/10.1016/S22138587(16)30100-0)

9 Beuschlein F, Mulatero P, Asbach E, Monticone S, Catena C, Sechi LA \& Stowasser M. The SPARTACUS Trial: controversies and unresolved issues. Hormone and Metabolic Research 201749 936-942. (https://doi. org/10.1055/s-0043-120524)

10 Williams TA, Burrello J, Sechi LA, Fardella CE, Matrozova J, Adolf C, Baudrand R, Bernardi S, Beuschlein F, Catena C, et al. Computed tomography and adrenal venous sampling in the diagnosis of unilateral primary aldosteronism. Hypertension 201872 641-649. (https://doi.org/10.1161/HYPERTENSIONAHA.118.11382)

Received in final form 24 March 2019

Accepted 9 May 2019 